\title{
Status of the novel CORSIKA 8 air shower simulation framework
}

\section{A. Augusto Alves $\mathrm{Jr}^{a, *}$ on behalf of the CORSIKA 8 Collaboration}

(a complete list of authors can be found at the end of the proceedings)

${ }^{a}$ Institut für Astroteilchenphysik - Karlsruher Institut für Technologie Karlsruhe, Germany

E-mail: augusto.alvesjunior@kit.edu

The CORSIKA 8 project is an international collaboration of scientists working together to implement a modern, flexible, robust and efficient framework for the simulation of ultra-high energy secondary particle cascades in matter. While the main application is for simulating cosmic ray air showers, the project aim to useful to other problems in astro(particle), particle and nuclear physics. Besides a comprehensive and state-of-the-art collection of physics models and algorithms relevant for the field, interfaces to deployment modern multicore processors and hardware accelerators (e.g. GPU) are also planned. This contribution presents the status and roadmap of the CORSIKA 8 project.

$37^{\text {th }}$ International Cosmic Ray Conference (ICRC 2021)

July 12th - 23rd, 2021

Online - Berlin, Germany

\footnotetext{
${ }^{*}$ Presenter
} 


\section{Introduction}

The legacy CORSIKA air shower simulation program[1] has been the de facto common reference frame for a vast class of measurements and studies in astroparticle physics for almost 30 years [2], making it possible to perform accurate comparative, incremental and progressive research in the field.

The CORSIKA 8 project[3] is basically the follow-up on this approach and aims to port and to implement it into a modern computing fashion. The effort is fundamentally community-driven and bundles modern concepts for computing with the goal for the most comprehensive and flexible physics description for the simulation of particle cascades. It will bring novel modularity to enable optimal and fine-tuned modeling as well as future developments. Among other planned features, it will be possible to better relate the importance of model parameter uncertainties on observables of interest and to incorporate improvements in physics related algorithms, where it is applicable. Concomitantly, a significant effort is being devoted to deploy concepts and techniques connected with modern high-performance computing through robust and user-friendly interfaces. For example, the need to balance high performance goals, with portability and modularity lead to the adoption of $\mathrm{C}++$ as the basic language of the framework.

In these proceedings, the key features of CORSIKA 8, as well as some very basic comparison of simulation results, are discussed.

\section{Scientific context and open problems}

The understanding of the formation of secondary particle cascades due to the interaction of high energy particles with the matter is a central problem in astroparticle physics. Indeed, these phenomena occur in many astrophysically relevant contexts, producing gamma-ray and neutrino emissions. When happening in Earth's atmosphere, the penetration of high energy cosmic-rays produces extensive air shower cascades (EAS), which are then observed by experiments.

The precise interpretation of the corresponding experimental data in terms of fundamental properties of the matter and the primary cosmic ray particles is crucial for understanding many aspects of the nature of the physics phenomena in the surround universe. As the involved energies increase, the more relevant are the opportunities for a more precise measurement of the primary composition of cosmic rays and also for understanding how such particles interact.

The precise description of EAS is a very challenging endeavor. Hadron collisions must be described with good quality from hundreds of $\mathrm{TeV}$ down to $\mathrm{GeV}$, at center-of-mass energy. This, most importantly, includes the relatively poorly known forward phase space, which requires dedicated input from experiments at accelerators. Cascades develop in air, which is a mixture of light elements, making the modelling of nuclear effects very important. Electromagnetic interactions must consider the density-dependent high-energy Landau-Pomeranchuk-Migdal (LPM) effect [4-6] which suppresses cross-sections at high energies as well as density-corrections at low energies.

Astroparticle physics observatories depend on the modelling of EAS for their design, operation and also for their data analyses. This is most obvious for indirect cosmic ray experiments, regardless if they measure particles at ground, fluorescence light, Cherenkov light, radio emission, or something else (e.g. [7]). But also neutrino observatories require a very precise understanding of their 


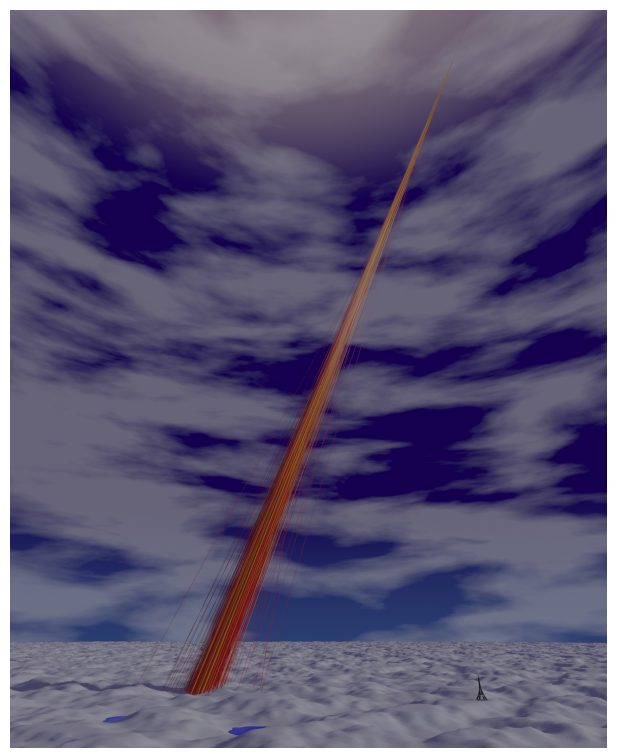

Figure 1: Example of a simulated proton shower at $E_{0}=10^{7} \mathrm{GeV}$ with CORSIKA 8 representing the propagation and interaction of hadrons and muons in earth magnetic field. Only particles above $50 \mathrm{GeV}$ are represented. Red are muons, yellow are hadrons.

atmospheric backgrounds, which are immense and extremely difficult to simulate. Finally, also the $\mathrm{PeV}$ gamma ray observatories need simulations for signal and background studies, sometimes even in a time-dependent and per-source setting[8].

Thus, basically all emission produced in EAS are used by experiments. CORSIKA 8 is the tool linking microscopic models to the final experimental observables. One of the big challenges is the better understanding of how uncertainties in input models affect observables.

In fig. 1 the 3D output of one EAS simulation performed with CORSIKA 8 is shown.

\section{Design overview and infrastructure}

As framework, CORSIKA 8 is designed to provide most, if not all, the infrastructure necessary to write concrete physics applications. The central loop involves a stack used for temporary storage of particles, a geometric transport routines and a list of processes that can lead to secondary-particle production or absorption. In terms of interpretation, the main goals driving the development summarized below.

- Modularity: It is a fundamental requirement that all algorithms and physics models are interfaced in a transparent, simple and modular way. Each physics process is provided as a module, with clear scope and having all necessary assumptions and parameters configurable. Among others conveniences, this design eases the implementation of new models, to extend or replace the delivered functionality. The different types of modules describing the physics of cascades are represented by $\mathrm{C}++$ class templates: ContinuousProcess, InteractionProcess, DecayProcess, SecondariesProcess, StackProcess, BoundaryCrossingProcess [9]. 
- Completeness: In order to perform accurate studies on systematic effects and generate comprehensive comparisons, CORSIKA 8 aims to provide interfaces for the most, if not all, approaches available in the literature to deal with a given problem.

- Robustness: In order to enable CORSIKA 8 to run over decades, a set of design choices has been made to ensure long-term stability. For example, the usage of plain old data (POD) C++ types is avoided in favor of statically typed constructs, e.g. "ContinuousProcessIndex" instead of just "int", particle codes are enumerated like in "Code code=Electron;". Statically typed units are used to express physics properties, like in "HEPEnergyType E0 = 10_GeV;" [10]. Such choices allow the compiler to perform extensive checks on the correctness of expressions, avoiding the most common mistakes.

- Performance: Specific attention is devoted to provide efficient implementations for core algorithms and modules. In particular, extensive support for modern multithread architectures, like multicore CPUs and GPUs is planned.

The CORSIKA 8 framework deploy a set of innovative and stat-of-the-art computing techniques and programming patterns to deal with pseudorandom number generation, track the history of secondary cascades, output and storage data. These and other implementation details are discussed in [11-13].

\section{Physics modules}

In terms of physics related features, CORSIKA 8 is not yet complete. Currently, available event generators for hadron collisions are SIBYLL2.3d [14], QGSJetII.04 [15], PYTHIA 8 [16] and UrQMD [17]. The latter is used at "low" energies (typically below around $100 \mathrm{GeV}$ ), while the former two can go up to the highest energies. The electromagnetic interactions and continuous/radiative energy losses of leptons are simulated with PROPOSAL [18-20]. Decays are currently delegated to PYTHIA 8 or SIBYLL. Charged particles moving in magnetic fields are tracked using a leap-frog algorithm. The region of interest, containing an environment potentially filled with different media is described using a custom geometry set of functionality, allowing the definition of different materials on large scales, to model situations like showers in the atmosphere which penetrate and get detected inside an ice shield at the South Pole. Thanks to the clear and simple interfaces, further physics modules can be easily added as it become available.

\section{Examples and preliminary results,}

The functionality implemented in CORSIKA 8 , albeit incomplete, allows the usage of the framework to perform a range of useful calculations, such as validation studies. It also provides insights for new types of research that maybe were not feasible before.

More specifically, the available physics modules for the hadronic and muonic shower components $[13,21]$ can be used for performing cross-validation with other air shower simulation codes, including legacy CORSIKA versions. For example, the fig. 2a shows the comparison of the energy spectra of secondary particles reaching the ground as obtained from simulations with CORSIKA 8 , 


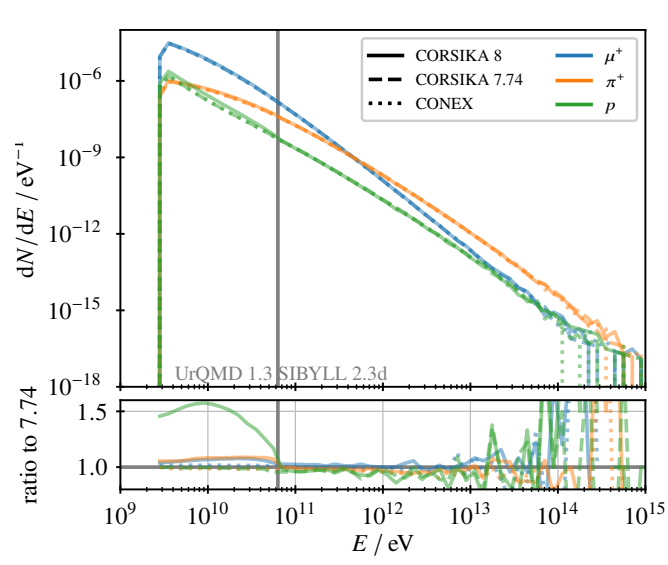

(a) Energy spectra of $\mu^{+}, \pi^{+}$and $p$

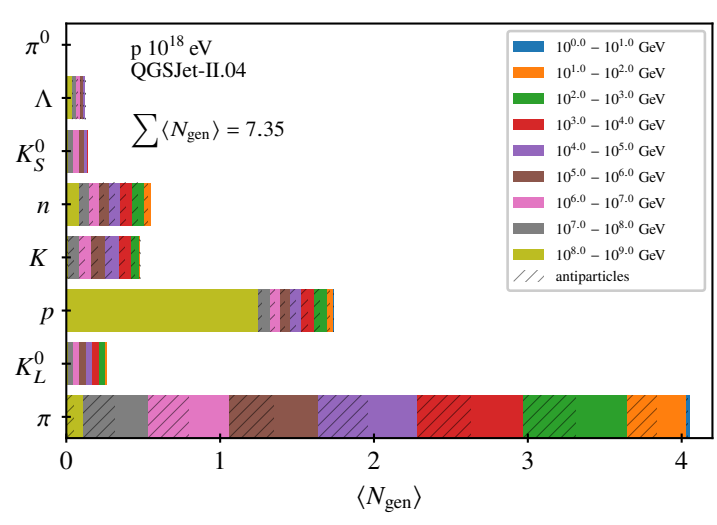

(b) Average number of muon ancestor

Figure 2: On the left, the energy spectra of $\mu^{+}, \pi^{+}$and $p$ of $1 \times 10^{17} \mathrm{eV}$ vertical proton shower, averaged over 400 events. On the right, the average number of muon ancestor projectile generations by particle species and energy.

CORSIKA 7.74 and CONEX [22] using the same parameters and interaction models in all three codes (vertical proton primary of $10^{17} \mathrm{eV}$, SIBYLL 2.3d and UrQMD 1.3 for hadronic interactions above and below $63.1 \mathrm{GeV}$, respectively). A good agreement is observed in the high energy regime. The low energy region exhibits some significant differences in the spectra of nucleons, which are attribute to differences in the interfaces to the event generator. The impact on the muon spectra, however, is small. First detailed results of such comparisons have been published elsewhere [23] and further studies are ongoing.

The fig. $2 b$ represents a first application using the cascade history tracking, recently implemented and lengthily described in $[12,13]$, the ancestry of projectiles that finally lead to a muon on ground[12]. At energies between the primary energy $\left(10^{18} \mathrm{eV}\right)$ and down to about a decade less, nucleon interactions dominate, with only a small fraction being antinucleons. Especially the impact of the primary proton is visible as it is the unique particle in the "zeroth generation" of all shower particles and therefore contributes 1 to $\left\langle N_{\text {gen }}\right\rangle$ in the highest energy bin. At lower energies $\left(\lesssim 10^{17} \mathrm{eV}\right.$ ), pion interaction are the main driver of the hadronic cascade and each decade in energy contributes roughly equally to the number of generations until around several $10 \mathrm{GeV}$ they decay into muons and almost no pion interacts again below $10 \mathrm{GeV}$.

Concomitantly, dedicated modules deploying efficient computing techniques, like GPU based acceleration and vectorization, are under development for calculation of Cherenkov and radio emissions[24-27]. The fig. 3b shows an example of Cherekov light emission simulation in GPU, calculated at ground level for a shower produced by a $1 \mathrm{TeV}$ gamma ray. The fig. 3a represents the pulse produced by radio using both algorithms CoREAS and ZHS running at CORSIKA 8, by a vertical electron shower of $100 \mathrm{TeV}$, on a layered atmosphere with a uniform refractive index of 1.000327 and a geomagnetic field $50 \mu \mathrm{T}$ in the $\mathrm{x}$-direction. The antenna is placed at $200 \mathrm{~m}$ from the shower core. 


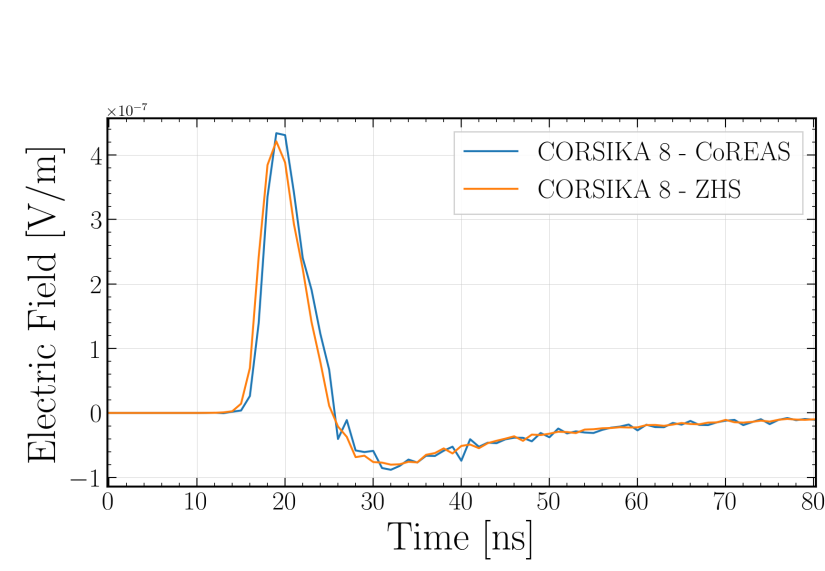

(a) Radio pulse

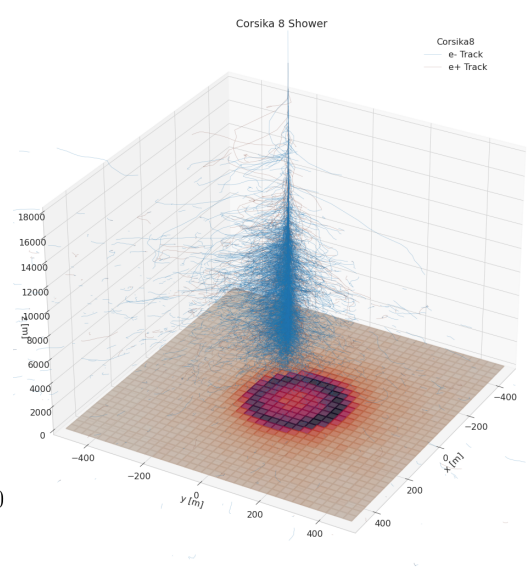

(b) Cherenkov light is calculated with GPU

Figure 3: On the left, a pulse produced by radio using both algorithms CoREAS and ZHS, by a vertical electron shower of $100 \mathrm{TeV}$. On the right, the Cherenkov light at ground level simulated in CORSIKA 8 using GPU.

\section{Summary and roadmap}

CORSIKA 8 framework, a novel high-performance computing platform for simulations of secondary particle cascades in astroparticle physics has been described. The framework is written in $\mathrm{C}++17$ and planned for supporting modern hardware architectures like multicore CPU and accelerators like GPUs and FPGAs, for running suited sub-problems with increased performance.

A first pre-release version is available for tests, validation and development [28]. This includes physics models, geometry, environment/media, output and configuration. Such a version should be used by the community for extensive tests, in order to provide the feedback needed for refinements and corrections, leading to a first full physics production release. It is foreseen that the setup as an open-source community project will support the goal for continued development over a very extended period of time.

\section{Acknowledgments}

The authors acknowledge support by the High Performance and Cloud Computing Group at the Zentrum für Datenverarbeitung of the University of Tübingen, the state of Baden-Württemberg through bwHPC and the German Research Foundation (DFG) through grant no. INST 37/9351 FUGG.

\section{References}

[1] D. Heck, J. Knapp, J.N. Capdevielle, G. Schatz and T. Thouw, CORSIKA: A Monte Carlo code to simulate extensive air showers, Tech. Rep. FZKA-6019, Forschungszentrum Karlsruhe (1998), DOI.

[2] H.J. Gils, D. Heck, J. Oehlschlaeger, G. Schatz, T. Thouw and A. Merkel, A MULTITRANSPUTER SYSTEM FOR PARALLEL MONTE CARLO SIMULATIONS OF EXTENSIVE AIR SHOWERS, Comput. Phys. Commun. 56 (1989) 105. 
[3] R. Engel, D. Heck, T. Huege, T. Pierog, M. Reininghaus, F. Riehn et al., Towards a Next Generation of CORSIKA: A Framework for the Simulation of Particle Cascades in Astroparticle Physics, Comput. Softw. Big Sci. 3 (2019) 2 [1808. 08226].

[4] A.B. Migdal, Bremsstrahlung and pair production in condensed media at high-energies, Phys. Rev. 103 (1956) 1811.

[5] L.D. Landau and I. Pomeranchuk, Electron cascade process at very high-energies, Dokl. Akad. Nauk Ser. Fiz. 92 (1953) 735.

[6] L.D. Landau and I. Pomeranchuk, Limits of applicability of the theory of bremsstrahlung electrons and pair production at high-energies, Dokl. Akad. Nauk Ser. Fiz. 92 (1953) 535.

[7] R. Smida et al., Observation of microwave emission from extensive air showers with CROME, EPJ Web Conf. 53 (2013) 08010.

[8] E. Santos for AUGER Collaboration, Monte Carlo simulations for the Pierre Auger Observatory using the VO Auger grid resources, in Proceedings of 37th International Cosmic Ray Conference (ICRC 2021) [29], https://pos.sissa.it/395/232/.

[9] M. Reininghaus and R. Ulrich, CORSIKA 8 - Towards a modern framework for the simulation of extensive air showers, EPJ Web Conf. 210 (2019) 02011 [1902 . 02822].

[10] CORSIKA 8 collaboration, Technical Foundations of CORSIKA 8: New Concepts for Scientific Computing, PoS ICRC2019 (2020) 236.

[11] A.A. Alves Junior, A. Poctarev and R. Ulrich, Counter-based pseudorandom number generators for CORSIKA 8: A multi-thread friendly approach, in Proceedings of 25th International Conference on Computing in High Energy and Nuclear Physics (CHEP 2021) [30], https://indico.cern.ch/event/948465/contributions/4324156/.

[12] R. Ulrich, A.A. Alves Junior, M. Reininghaus, R. Prechelt and A. Schmidt, CORSIKA 8 - A novel high-performance computing tool for particle cascade Monte Carlo simulations, in Proceedings of 25th International Conference on Computing in High Energy and Nuclear Physics (CHEP 2021) [30], https://indico.cern.ch/event/948465/contributions/4324165/.

[13] M. Reininghaus for CORSIKA 8 Collaboration, Air shower genealogy for muon production, in Proceedings of 37th International Cosmic Ray Conference (ICRC 2021) [29], https://pos.sissa.it/395/463/.

[14] F. Riehn, R. Engel, A. Fedynitch, T.K. Gaisser and T. Stanev, Hadronic interaction model Sibyll 2.3d and extensive air showers, Phys. Rev. D 102 (2020) 063002 [1912.03300].

[15] S. Ostapchenko, Monte Carlo treatment of hadronic interactions in enhanced Pomeron scheme: I. QGSJET-II model, Phys. Rev. D 83 (2011) 014018 [1010 . 1869].

[16] T. Sjöstrand, S. Ask, J.R. Christiansen, R. Corke, N. Desai, P. Ilten et al., An introduction to PYTHIA 8.2, Comput. Phys. Commun. 191 (2015) 159 [1410.3012]. 
[17] M. Bleicher et al., Relativistic hadron hadron collisions in the ultrarelativistic quantum molecular dynamics model, J. Phys. G 25 (1999) 1859 [hep-ph/9909407].

[18] J.-M. Alameddine, J. Soedingrekso, A. Sandrock, M. Sackel and W. Rhode, PROPOSAL: A library to propagate leptons and high energy photons, J. Phys. Conf. Ser. 1690 (2020) 012021.

[19] M. Dunsch, J. Soedingrekso, A. Sandrock, M. Meier, T. Menne and W. Rhode, Recent Improvements for the Lepton Propagator PROPOSAL, Comput. Phys. Commun. 242 (2019) 132 [1809.07740].

[20] J.H. Koehne, K. Frantzen, M. Schmitz, T. Fuchs, W. Rhode, D. Chirkin et al., PROPOSAL: A tool for propagation of charged leptons, Comput. Phys. Commun. 184 (2013) 2070.

[21] R. Ulrich for CORSIKA 8 Collaboration, Hadron cascades in CORSIKA 8, in Proceedings of 37th International Cosmic Ray Conference (ICRC 2021) [29], https://pos.sissa.it/395/474/.

[22] T. Bergmann, R. Engel, D. Heck, N.N. Kalmykov, S. Ostapchenko, T. Pierog et al., One-dimensional Hybrid Approach to Extensive Air Shower Simulation, Astropart. Phys. 26 (2007) 420 [astro-ph/0606564].

[23] CORSIKA 8 collaboration, First results of the CORSIKA 8 air shower simulation framework, PoS ICRC2019 (2020) 399.

[24] N. Karastathis for CORSIKA 8 Collaboration, Simulations of radio emission from air showers with CORSIKA 8, in Proceedings of 37th International Cosmic Ray Conference (ICRC 2021) [29], https://pos.sissa.it/395/427/.

[25] J.M. Alameddine for CORSIKA 8 Collaboration, Electromagnetic Shower Simulation for CORSIKA 8, in Proceedings of 37th International Cosmic Ray Conference (ICRC 2021) [29], https://pos.sissa.it/395/428/.

[26] D. Baack for CORSIKA 8 Collaboration, GPU Accelerated optical light propagation in CORSIKA8, in Proceedings of 37th International Cosmic Ray Conference (ICRC 2021) [29], https://pos.sissa.it/395/705/.

[27] L. Arrabito, D. Parello, J. Bregeon, P. Langlois and G. Vasileiadis, A C++ Cherenkov photons simulation in CORSIKA 8, in Proceedings of 25th International Conference on Computing in High Energy and Nuclear Physics (CHEP 2021) [30], https://indico.cern.ch/event/948465/contributions/4324127/.

[28] https://gitlab.ikp.kit.edu/AirShowerPhysics/corsika.

[29] Proceedings of 37th International Cosmic Ray Conference (ICRC 2021), PoS ICRC2021, To appear.

[30] Proceedings of 25th International Conference on Computing in High Energy and Nuclear Physics (CHEP 2021), EPJ Web of Conf., To appear. 


\section{Full Authors List: CORSIKA 8 Collaboration}

Jean-Marco Alameddine ${ }^{b}$, Johannes Albrecht ${ }^{b}$, Jaime Alvarez-Muniz ${ }^{r}$, Antonio Augusto Alves $\mathrm{Jr}^{d}{ }^{\text {, Luisa Arrabito }}{ }^{a}$, Dominik Baack ${ }^{b}$, Konrad Bernlöhr $^{c}$, Marcus Bleicher ${ }^{o}$, Johan Bregeon ${ }^{s}$, Mathieu Carrere ${ }^{a}$, Hans Dembinski ${ }^{b}$, Hannah Elfner ${ }^{i}$, Dominik Elsässer ${ }^{b}$, Ralph Engel $^{d}$, Hu Fan ${ }^{p}$, Anatoli Fedynitch ${ }^{j}$, Dieter Heck ${ }^{d}$, Tim Huege ${ }^{d, e}$, Karl-Heinz Kampert ${ }^{k}$, Nikolaos Karastathis ${ }^{d}$, Lukas Nellen ${ }^{f}$, Maximilian Nöthe ${ }^{b}$, David Parello ${ }^{t}$, Tanguy Pierog ${ }^{d}$, Maria Pokrandt ${ }^{d}$, Anton Poctarev ${ }^{d}$, Remy Prechelt ${ }^{l}$, Maximilian Reininghaus $^{d, m}$, Wolfgang Rhode ${ }^{b}$, Felix Riehn ${ }^{h, r}$, Maximilian Sackel ${ }^{b}$, Alexander Sandrock ${ }^{u}$, Pranav Sampathkumar ${ }^{d}$, Michael Schmelling ${ }^{c}$, André Schmidt ${ }^{d}$, Günter Sigl ${ }^{n}$, Jan Soedingrekso ${ }^{b}$, Bernhard $\operatorname{Spaan}^{b}$, Donglian $\mathrm{Xu}^{q}$, Juan Ammerman-Yebra ${ }^{r}$ and Enrique $\mathrm{Zas}^{r}$

${ }^{a}$ Laboratoire Univers et Particules, Université de Montpellier 2, Montpellier, France, ${ }^{b}$ Experimentelle Physik 5, TU Dortmund, Dortmund, Germany, ${ }^{c}$ Max Planck Institute for Nuclear Physics, Heidelberg, Germany, ${ }^{d}$ Institute for Astroparticle Physics, Karlsruhe Institute of Technology, Karlsruhe, Germany, ${ }^{e}$ Astrophysical Institute, Vrije Universiteit Brussel, Brussels, Belgium ${ }^{f}$ National Autonomous University of Mexico, Mexico City, Mexico, ${ }^{g}$ Instituto de Tecnologías en Detección y Astropartículas, Buenos Aires, Argentina, ${ }^{h}$ Laboratory of Instrumentation and Experimental Particles, Lisbon, Portugal, ${ }^{i}$ Helmholtzzentrum für Schwerionenforschung, Darmstadt, Germany, ${ }^{j}$ Institute for Cosmic Ray Research, The University of Tokyo, Tokyo, Japan, ${ }^{k}$ Lehrstuhl für Astroteilchenphysik, Bergische Universität Wuppertal, Wuppertal, Germany, ${ }^{l}$ Department of Physics Astronomy, University of Hawai'i at Manoa, Honolulu, USA, ${ }^{m}$ Instituto de Tecnologías en Detección y Astropartículas, Buenos Aires, Argentina, ${ }^{n}$ II Institut für Theoretische Physik, Universität Hamburg, Hamburg, Germany, ${ }^{o}$ Johann-Wolfgang-Goethe-Universitaet, Frankfurt am Main, Germany, ${ }^{p}$ Peking University, Beijing, China, ${ }^{q}$ Tsung-Dao Lee Institute, Shanghai, China, ${ }^{r}$ Instituto Galego de Física de Altas Enerxías, Universidade de Santiago de Compostela, Santiago de Compostela, Spain, ${ }^{s}$ Laboratoire de Physique Subatomique et de Cosmologie, Grenoble, France, ${ }^{t}$ LIRMM, Univ Montpellier, CNRS, Montpellier, France, ${ }^{u}$ National Research Nuclear University, Moscow Engineering Physics Institute, Moscow, Russia. 